\title{
First-principles molecular dynamics simulations at solid-liquid interfaces with a continuum solvent
}

\author{
Verónica M. Sánchez, ${ }^{1}$ Mariela Sued, ${ }^{2}$ and Damián A. Scherlis ${ }^{1, a)}$ \\ ${ }^{1}$ Departamento de Química Inorgánica, Analítica y Química Física/INQUIMAE, Facultad de Ciencias \\ Exactas y Naturales, Universidad de Buenos Aires, Ciudad Universitaria, Pab. II, Buenos Aires C1428EHA, \\ Argentina \\ ${ }^{2}$ Instituto de Cálculo, Facultad de Ciencias Exactas y Naturales, Universidad de Buenos Aires, Ciudad \\ Universitaria, Pab. II, Buenos Aires C1428EHA, Argentina
}

(Received 5 March 2009; accepted 5 October 2009; published online 4 November 2009)

\begin{abstract}
Continuum solvent models have become a standard technique in the context of electronic structure calculations, yet no implementations have been reported capable to perform molecular dynamics at solid-liquid interfaces. We propose here such a continuum approach in a density functional theory framework using plane-wave basis sets and periodic boundary conditions. Our work stems from a recent model designed for Car-Parrinello simulations of quantum solutes in a dielectric medium [D. A. Scherlis et al., J. Chem. Phys. 124, 074103 (2006)], for which the permittivity of the solvent is defined as a function of the electronic density of the solute. This strategy turns out to be inadequate for systems extended in two dimensions: the dependence of the dielectric function on the electronic density introduces a new term in the Kohn-Sham potential, which becomes unphysically large at the interfacial region, seriously affecting the convergence of the self-consistent calculations. If the dielectric medium is properly redefined as a function of the atomic coordinates, a good convergence is obtained and the constant of motion is conserved during the molecular dynamics simulations. The Poisson problem is solved using a multigrid method, and in this way Car-Parrinello molecular dynamics simulations of solid-liquid interfaces can be performed at a very moderate computational cost. This scheme is employed to investigate the acid-base equilibrium at the $\mathrm{TiO}_{2}$-water interface. The aqueous behavior of titania surfaces has stimulated a large amount of experimental research, but many open questions remain concerning the molecular mechanisms determining the chemistry of the interface. Here we make an attempt to answer some of them, putting to the test our continuum model. () 2009 American Institute of Physics. [doi:10.1063/1.3254385]
\end{abstract}

\section{INTRODUCTION}

The structure and the reactivity of solid surfaces have become major subjects of study in chemistry and condensed matter physics and are at the core of much of the research conducted in materials science. In this context, electronic structure methods, in particular density functional theory (DFT) calculations, have played a fundamental role in the interpretation and the validation of the data coming from the different surface spectroscopies and microscopies, often providing new insights on top of those detaching from the available experimental techniques. ${ }^{1}$ Throughout the past two decades a large number of DFT simulations have been reported on metallic and semiconducting surfaces, contributing precious information concerning atomic and electronic structure, thermodynamics, and reactivity. ${ }^{2-4}$ With very few exceptions, these simulations considered a slab in the gas phase. For a broad range of applications, however, the relevant phenomena occur in the presence of a liquid phase, as is often the case in processes related to electrochemistry and catalysis. The realization of liquid phase DFT simulations is therefore a much pursued objective-especially when many of the standard X-ray techniques such as XPS or SAXS are

${ }^{a)}$ Electronic mail: damian@qi.fcen.uba.ar. unsuited to provide atomic scale information in solutionbut the inclusion of the solvent considerably increases the cost of first-principles calculations of periodic surfaces and is therefore a rather uncommon practice. ${ }^{5}$

In an explicit solvation approach, the vacuum space in the simulation box is filled with solvent molecules. The subsequent growth in the size of the system is in part responsible for the increased computational expense, but more importantly, there is the fact that a static picture is a very poor representation of the liquid state. Any solute admits a huge number of possible configurations for the solvent molecules around it, associated with multiple local minima, the net solvation effect arising from a weighted average of all these. ${ }^{6} \mathrm{~A}$ geometry optimization would lead to a solid or glassy phase corresponding to one of these minima in the multidimensional potential energy surface, resulting in a dielectric screening typically different from the static limit observed in the liquid state. Hence, to capture the solvation effect, it is necessary to perform extensive statistical sampling involving either lengthy molecular dynamics or Monte-Carlo simulations. Alternatively, it is possible to resort to the so-called continuum (or implicit solvent) models in which the solvent molecules are replaced by a dielectric medium surrounding the solute and exhibiting the static screening of the 
solution. ${ }^{7,8}$ In this way, the polarization induced by the solvent is introduced in an averaged fashion, and the cost of the computation gets closer to the corresponding cost in vacuum by drastically reducing the number of degrees of freedom. On the other hand, the representation of the solvent structure is omitted, disregarding any possible solute-solvent specific interactions. Still, to overcome this problem, all or part of the first solvation shells can be included explicitly, with the dielectric medium extending beyond the limits of this cluster comprising the solute plus a few solvent molecules. In any case, the continuum model has a long tradition in quantum chemistry and has proven to be reliable and efficient to extract properties in solution of a large variety of molecular systems. ${ }^{6-9}$

In recent years a small number of implementations of the continuum model have been proposed in the context of DFT, plane-wave basis sets, and the Car-Parrinello method. ${ }^{10-13}$ To the best of our knowledge, none of them have been employed in molecular dynamics simulations of periodic surfaces. ${ }^{14}$ Here we revise the electrostatic continuum model of Fattebert and Gygi, ${ }^{11,13,15}$ proposing a new definition for the dielectric function to make the model suitable for the treatment of periodic slabs. The original formulation, in which the permittivity of the solvent is determined by the charge density of the solute, has proven to be successful in the simulation of molecular and ionic solutes ${ }^{11,13}$ and of extended systems such as polymers with periodicity in one dimension. ${ }^{16}$ In the case of solid surfaces, however, the convergence to the electronic ground state turns out to be impaired. In the sections that follow, we attribute this failure to a term in the Kohn-Sham potential originating in the dependence of the dielectric constant on the electronic density, and to circumvent this issue, we redefine the permittivity as a function of the atomic positions. In this way the smooth electronic convergence is restored, along with a good conservation of the total energy in the molecular dynamics runs. The electrostatic problem in the presence of the dielectric medium is efficiently addressed with a multigrid method, ${ }^{17-19}$ which solves the Poisson equation in real space. Using this scheme it is possible to carry out Car-Parrinello molecular dynamics simulations of solid-liquid interfaces at a computational cost exceeding by just a small factor the one corresponding to vacuum. We employ this approach to investigate the proton exchange at the anatase-water interface, which is a key process in the acid-base equilibrium of $\mathrm{TiO}_{2}$ surfaces. The ubiquity of titania in solid-liquid applications has incited the emergence of empirical models to assess the protonation and the dissociation of terminal groups on the $\mathrm{TiO}_{2}$ surface in aqueous environments. ${ }^{20,21}$ These simple models have been broadly used among experimentalists for the interpretation of their data, but a molecular level description that accounts for the effect of structure has not been established. In this context, the present simulations intend to provide a firstprinciples glance of the microscopic mechanisms governing the chemistry of this interface.

\section{MODEL AND METHODOLOGICAL DISCUSSION}

\section{A. About the present implementation and the computational parameters}

This model has been implemented in the public domain Car-Parrinello parallel code included in the QUANTUMESPRESSO package ${ }^{22}$ based on DFT, periodic boundary conditions, plane-wave basis sets, and pseudopotentials to represent the ion-electron interactions. All calculations reported in this work, unless otherwise noted, have been performed using the PW91 exchange-correlation functional ${ }^{23}$ in combination with Vanderbilt ultrasoft pseudopotentials. ${ }^{24}$ The Kohn-Sham orbitals and charge density were expanded in plane waves up to a kinetic energy cutoff of 25 and $200 \mathrm{Ry}$, respectively. Periodic slabs of four layers width representing the (101) surface of the anatase structure were computed using gamma-point sampling in supercells of size 10.24 $\times 7.56 \times 17.82 \AA^{3}$. For finite temperature (not damped) Car-Parrinello molecular dynamics simulations, an electronic mass of 400 a.u. and a time step of 0.17 fs were adopted. During geometry relaxations and dynamics, all atoms were allowed to move, with the exception of those belonging to the two inner layers, which were fixed in their bulk positions.

\section{B. The continuum solvation model}

Within the continuum approach, the solvent is represented as a dielectric medium surrounding a quantummechanical solute confined in a cavity. In particular, we consider the polarizable continuum model in which the dielectric medium and the electronic density respond to the field of each other in a self-consistent fashion. This interaction provides the electrostatic part of the solvation free energy $\Delta G_{\mathrm{el}}$, which is the dominant contribution for polar and charged solutes. The cavitation energy $\Delta G_{\text {cav }}$ is defined as the work involved in creating the appropriate cavity inside the solution in the absence of solute-solvent interactions. ${ }^{7}$ Electrostatic, cavitation, and dispersion-repulsion effects are modeled as separate contributions, ${ }^{7}$ and the free energy of solvation is regarded as the sum of these three terms $\left(\Delta G_{\mathrm{sol}}=\Delta G_{\mathrm{el}}\right.$ $\left.+\Delta G_{\text {cav }}+\Delta G_{\text {dis-rep }}\right)$. Thermal and pressure dependent terms can also be included but are usually negligible. We note that this decomposition is inherent to the model, being $\Delta G_{\text {sol }}$ the only measurable quantity.

\section{Previous implementation}

The starting point for this work is the implementation reported in Ref. 13. In the following paragraphs we revisit those aspects of the preceding version that are essential to the present development. First, we note that $\Delta G_{\mathrm{el}}$ and $\Delta G_{\mathrm{cav}}$ are considered explicitly, while $\Delta G_{\text {dis-rep }}$, less relevant for solutes of moderate size, is largely seized as part of the electrostatic term by virtue of the parametrization. The electrostatic interaction between the dielectric and the solute is calculated as proposed by Fattebert and Gygi, ${ }^{11,15}$ who defined the permittivity $\epsilon$ of the solvent as a function of the electronic density 
$\rho$. Within a pseudopotential framework and in periodic boundary conditions, the Kohn-Sham energy functional ${ }^{25}$ can be written as

$$
\begin{aligned}
E[\rho]= & T[\rho]+E_{\mathrm{xc}}[\rho]+\frac{1}{2} \int \phi(\mathbf{r}) \rho_{\mathrm{tot}}(\mathbf{r}) d \mathbf{r} \\
& +\sum_{I<J} \frac{Z_{I} Z_{J}}{R_{I J}} \operatorname{erfc}\left(\frac{R_{I J}}{\sqrt{\left(R_{I}^{c}\right)^{2}+\left(R_{J}^{c}\right)^{2}}}\right) \\
& -\frac{1}{\sqrt{2 \pi}} \sum_{I} \frac{Z_{I}^{2}}{R_{I}^{c}}+E_{\mathrm{ps}}[\rho]
\end{aligned}
$$

where $T[\rho]$ corresponds to the kinetic energy of the electrons and $E_{\mathrm{xc}}[\rho]$ to the exchange-correlation energy. The last four terms on the right hand side account for the total electrostatic energy in a periodic crystal, gathering all Coulombic interactions involving electrons and nuclei, only omitting for simplicity the nonlocal part of the pseudopotential (for a detailed derivation, see Ref. 26 or the Appendix of Ref. 13). The electrostatic formulation in Eq. (1) arises from the Ewald sum of point charges, which requires to introduce the ionic densities $\rho_{I}\left(\mathbf{r}-\mathbf{R}_{\mathbf{I}}\right)$ consisting of Gaussian distributions of negative sign that integrate to $Z_{I}$, the total charge of the pseudoion: $\rho_{I}\left(\mathbf{r}-\mathbf{R}_{\mathbf{I}}\right)=-\left[Z_{I} /\left(R_{I}^{c}\right)^{3}\right] \pi^{-3 / 2}$ $\times \exp \left(-\left[\left|r-R_{I}\right|^{2} /\left(R_{I}^{c}\right)^{2}\right]\right)$, with $R_{I}^{c}$ being the width of the Gaussian associated with the site $I$.

The third term on the right is conventionally called the Hartree energy,

$$
E_{H}=\frac{1}{2} \int \phi(\mathbf{r}) \rho_{\mathrm{tot}}(\mathbf{r}) d \mathbf{r},
$$

where $\rho_{\text {tot }}$ is just the sum of the electronic plus the ionic densities, $\rho_{\text {tot }}(\mathbf{r})=\rho(\mathbf{r})+\Sigma_{I} \rho_{I}\left(\mathbf{r}-\mathbf{R}_{\mathbf{I}}\right)$, while the electrostatic potential $\phi[\rho]$ is the solution to the Poisson equation in vacuum,

$$
\nabla^{2} \phi(\mathbf{r})=-4 \pi \rho_{\text {tot }}(\mathbf{r})
$$

In the presence of a dielectric continuum with a permittivity $\epsilon[\rho]$, the Poisson equation becomes

$$
\nabla \cdot(\epsilon[\rho] \nabla \phi(\mathbf{r}))=-4 \pi \rho_{\mathrm{tot}}(\mathbf{r}) .
$$

Using Eq. (3), the formula for the Hartree energy $E_{H}$ can be integrated by parts to yield

$$
E_{H}=\frac{1}{8 \pi} \int \epsilon[\rho](\nabla \phi(\mathbf{r}))^{2} d \mathbf{r} .
$$

The functional derivative of $E_{H}$ with respect to the charge density is added to the other contributions of the energy (the exchange-correlation, the local, and the nonlocal parts of the pseudopotentials) to set up the Kohn-Sham potential $V^{\mathrm{KS}}[\rho]$,

$$
\begin{aligned}
& \frac{\delta E_{H}}{\delta \rho}(\mathbf{r})=\phi(\mathbf{r})+V_{\epsilon}(\mathbf{r}), \\
& V_{\epsilon}(\mathbf{r})=-\frac{1}{8 \pi}(\nabla \phi(\mathbf{r}))^{2} \frac{\delta \epsilon}{\delta \rho}(\mathbf{r}) .
\end{aligned}
$$

We provide the derivation of Eqs. (5) and (6) in Appendix A since it is not given in any of the previous references. The dielectric medium and the electronic density respond selfconsistently to each other through the dependence of $\epsilon$ on $\rho$ and vice versa.

In quantum chemistry continuum models as PCM,,${ }^{9,27}$ the dielectric constant $\epsilon$ is taken to be one inside the cavity and a fixed value outside. For molecular dynamics applications, such a discontinuity needs to be removed to calculate accurately the analytic derivatives of the potential with respect to the ionic positions. Besides, in the particular case of planewave implementations based on real space grids, a smoothly varying dielectric function is more appropriate for numerical reasons. Fattebert and Gygi proposed the following smoothed step function for the dielectric:

$$
\epsilon(\rho(\mathbf{r}))=1+\frac{\epsilon_{\infty}-1}{2}\left(1+\frac{1-\left(\rho(\mathbf{r}) / \rho_{0}\right)^{2 \beta}}{1+\left(\rho(\mathbf{r}) / \rho_{0}\right)^{2 \beta}}\right) .
$$

This function asymptotically approaches $\epsilon_{\infty}$ (the permittivity of the bulk solvent) in regions of space where the electron density is low and one in those regions where it is high (outside the solvation cavity). The parameter $\rho_{0}$ is the density threshold determining the cavity size, whereas $\beta$ modulates the smoothness of the transition from $\epsilon_{\infty}$ to 1 .

On the other hand, the cavitation energy is computed separately as the product between the surface tension of the solvent and the area of the cavity. In this implementation this term remains unchanged; details about the calculation of $\Delta G_{\text {cav }}$ can be found in Ref. 13 .

\section{Calculation of periodic slabs: The problem in the convergence}

When the scheme described above is applied on structures extended in two dimensions, it typically fails to achieve self-consistency. We found that the electrons heat up during the Car-Parrinello relaxation of the wave function, causing the total energy to diverge. In other cases the kinetic energy of the electrons is observed to decrease at the beginning but thereafter experiences irregular oscillations without ever reaching zero. Even if convergence is enforced through the use of a stringent algorithmic strategy, the solution obtained is not reliable, e.g., it depends on the starting conditions or the damping parameters.

The source of this erratic behavior can be tracked to the inclusion of the term $V_{\epsilon}$ in the Kohn-Sham potential. This term, defined in Eq. (6), arises from the dependence of the permittivity on the electron density. The impact of this contribution on the convergence of a Car-Parrinello electronic relaxation is evinced by the fact that an acceptable dynamics is recovered if $V_{\epsilon}$ is neglected. ${ }^{28}$ In particular, this pathology has been characterized for the anatase-water structure described in Sec. III, but other systems are affected in a similar way. The reason for the instability associated with $V_{\epsilon}$ can be somehow appreciated in the two dimensional plot in Fig. 1, which displays the variation in $V_{\epsilon}$ in the $z$-direction (the one perpendicular to the surface) at fixed $x$ and $y$. These coordinates have been chosen in coincidence with the position of a $\mathrm{Ti}$ atom of the surface. The same graph shows the total Kohn-Sham potential, excluding $V_{\epsilon}$, so that the relative contribution of this term can be clearly seen. The values are 


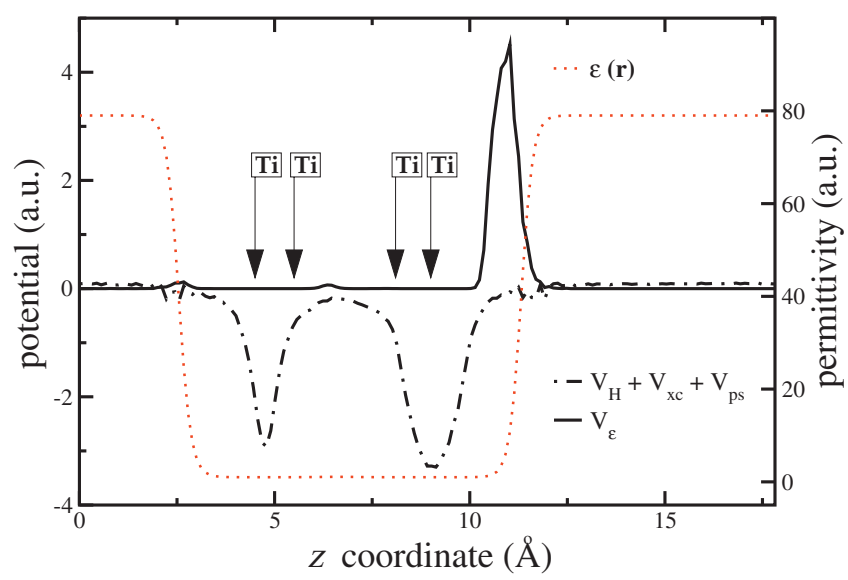

FIG. 1. Variation in $V_{\epsilon}$ and of the effective potential along the $z$-direction (perpendicular to the slab) at a selected point on the $x-y$ plane, corresponding to the position of one of the exposed Ti atoms of the surface. The dotted line represents the permittivity referenced on the right axis. The arrows point to the approximate location of each of the titanium layers.

exceptionally large at the solid-liquid boundary, even larger than any of the other contributions to the effective potential. Referenced on the right axis, the dielectric function $\epsilon[\rho]$ is also shown. Since $V_{\epsilon}$ depends on $\partial \epsilon / \partial \rho$ [Eq. (6)], the peaks occur at the region where the charge density decays abruptly, producing a rapid variation in $\epsilon[\rho]$ from the value in the solid to the value in the solvent. Adopting the model function of Eq. (7), we have

$$
\frac{\partial \epsilon}{\partial \rho}(\mathbf{r})=\frac{1-\epsilon_{\infty}}{\rho_{0}} \frac{2 \beta\left(\rho(\mathbf{r}) / \rho_{0}\right)^{2 \beta-1}}{\left(1+\left(\rho(\mathbf{r}) / \rho_{0}\right)^{2 \beta}\right)^{2}} .
$$

This expression goes to zero when $\rho(\mathbf{r}) \gg \rho_{0}$ or $\rho(\mathbf{r}) \ll \rho_{0}$ and is dominated by $1 / \rho_{0}$ otherwise. $^{29}$ Then, the extreme values of $V_{\epsilon}$ at the solid-liquid interface ultimately originate in the magnitude of the density threshold $\rho_{0}$. Unfortunately, this parameter may not be freely tuned but is set in combination with $\beta$ to fit the experimental data, and no reasonable choice of $\left(\rho_{0}, \beta\right)$ can be made as to prevent the blowup of $V_{\epsilon}$. Alternative model functions to represent the dielectric were considered, e.g., with $\epsilon[\rho]$ exhibiting a Gaussian or trigonometric decay with $\rho$. None of these functions entailed any significant improvement, as far as all of them have in common a sudden change associated with the transition from 1 to $\epsilon_{\infty}$, which redounds in large values of $\partial \epsilon / \partial \rho$ at the interface. The transition can be smoothed enough as to avoid the sharp peaks in $V_{\epsilon}$, but in doing so the solvation effect is ruined. In other words, we found that a simple redefinition of the dielectric function does not suffice to recover numerical stability; we were unable to find a functional form for $\epsilon$ providing at the same time good convergence and a realistic solvation energy. The discussed behavior is a consequence of the dependence of $\epsilon$ on $\rho$, irrespective of the kind of function chosen to model the dielectric. It should be noticed that the inclusion of $V_{\epsilon}$ does not appear to disturb the convergence or the energy conservation in the case of finite systems, neither in the case of polymers (extended in one dimension). ${ }^{16}$ It is seemingly because of the bidimensional symmetry of periodic surfaces that $V_{\epsilon}$ turns out to spoil the Car-Parrinello dynamics.

\section{E. A position-dependent formulation}

It can be argued that convergence fails because the noise in the representation of the almost-divergent potential does not allow the system to make steps in the correct (minimum) direction. In such a case, the implementation of denser grids should bring some relief to the computational convergence. Trial runs with the number of mesh points increased up to $50 \%$ on each direction-meaning an increment of more than three times in the density of the real space grids-however, did not improve the situation in any noticeable way. This suggests exceedingly high energy cutoffs should be used to restore convergence.

In the absence of a dielectric or for a dielectric defined independently of the charge density, the functional derivative of $E_{H}$ with respect to $\rho$ turns out to be equal to the electrostatic potential $\phi[\rho]$ (see Ref. 30). The additional term $V_{\epsilon}$ emerges from the dependence of $E_{H}$ on $\epsilon$, which is a function of $\rho$, and thus, the instability of the model emanates from the self-consistency between the charge density and the dielectric. A possible alternative would be to use instead a nonself-consistent or "fake" density since the role of the charge density in this context is simply to shape the dielectric medium. This solution has been adopted in recent work applying the model of Fattebert and Gygi to electrochemical interfaces, where the dependence of the potential on the dielectric function was omitted. ${ }^{31}$ Such an approach, however, does not conserve the constant of motion in molecular dynamics simulations. Yet another path to relax the explicit dependence of the potential on the permittivity would be to define $\epsilon$ as a function of the atomic coordinates, which is the usual strategy in quantum chemistry methods. The idea of a dielectric determined by the atomic positions may be less attractive from a physical viewpoint: the size and shape of the cavity depend on the identity of the atoms only and is not modulated by the electronic structure or the environment; the polarization of the solvent is lost, and on top of these, many more parameters are needed-at least one per atom. In practice, however, it is possible to choose a dielectric function based on the molecular coordinates that closely reproduces the $\rho$-dependent solvation because the effect of the selfconsistency and the polarization of the solvent on $\Delta G_{\text {sol }}$ is quite minor.

In this way, we keep the expression for $\epsilon$ given in Eq. (7) but feed it with a dummy density $\gamma(\mathbf{r})$ determined by the ionic positions $\mathbf{R}_{\mathbf{I}}$,

$$
\gamma(\mathbf{r})=\sum_{I} e^{-\left(\left|\mathbf{r}-\mathbf{R}_{\mathbf{I}}\right|-R_{\mathrm{vdw}}^{I}\right)},
$$

where $R_{\mathrm{vdw}}^{I}$ is the van der Waals radius for the corresponding species. Hence the dielectric function takes the following form:

$$
\epsilon(\gamma(\mathbf{r}))=1+\frac{\epsilon_{\infty}-1}{2}\left(1+\frac{1-\gamma(\mathbf{r})^{2 \beta}}{1+\gamma(\mathbf{r})^{2 \beta}}\right) .
$$

Using this definition, the transition of $\epsilon(\gamma(\mathbf{r}))$ between 1 and $\epsilon_{\infty}$ is centered around the van der Waals radius. Aside from $R_{\mathrm{vdw}}^{I}$, which values are tabulated, $\beta$ is left as the only adjustable parameter to fit the experimental solvation ener- 


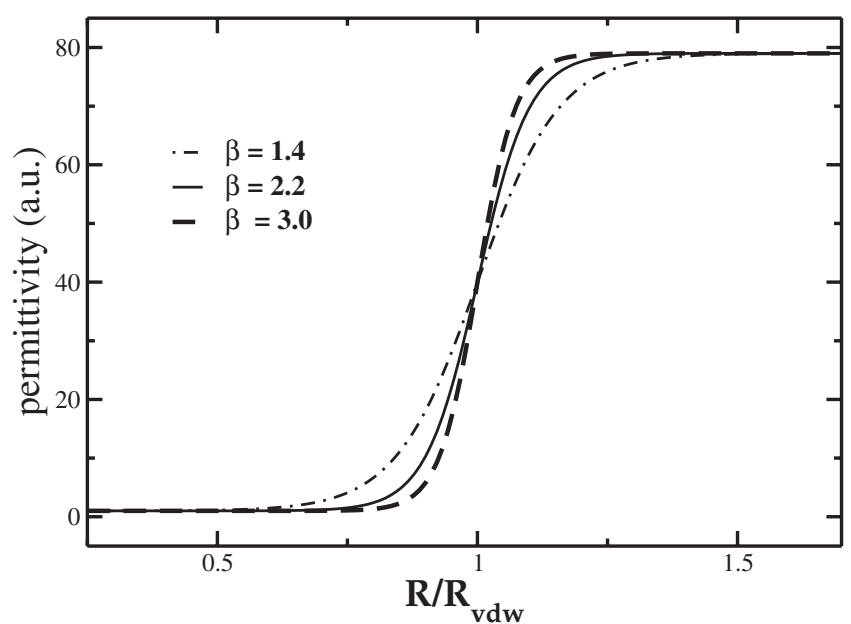

FIG. 2. The permittivity around an oxygen atom as a function of the distance for different values of the parameter $\beta$ according to the positiondependent dielectric defined in Eqs. (9) and (10). The transition, centered around the van der Waals radius, becomes sharper as $\beta$ is increased.

gies. Figure 2 shows the aspect of the dielectric function around an oxygen atom for different $\beta$. This parameter must be large enough to ensure most of the transition occurs within a small length window, but at the same time the numerical accuracy needs to be preserved, so there is an upper bound for $\beta$, which depends on the given grid size.

Within this framework, the electrostatic contribution to $V^{\mathrm{KS}}[\rho]$ is simply the electrostatic potential $\phi[\rho]$, and the stability of Car-Parrinello dynamics in periodic slabs is recovered. Table I presents, for several neutral and charged solutes, a comparison between the values of $\Delta G_{\text {sol }}$ obtained with the dielectric functions of Eqs. (7) and (10), respectively. The small differences proceed exclusively from $\Delta G_{\mathrm{el}}$ since $\Delta G_{\text {cav }}$ is the same in both cases $\left(\Delta G_{\text {sol }}=\Delta G_{\text {el }}+\Delta G_{\text {cav }}\right)$. As mentioned above, with a proper choice of $\beta$ the positiondependent dielectric is able to provide solvation energies in close agreement with the previous model and with experiments. The results shown correspond to $\beta=2.4$.

The explicit dependence of $\epsilon$ on the ionic positions involves a new contribution to the forces arising from the derivative of $E_{H}$ with respect to $\mathbf{R}_{\mathbf{I}}$, which must be taken into account to perform conservative molecular dynamics simulations. After some manipulation involving the use of Eqs. (3) and (4), this derivative can be expressed as follows (the full derivation is given in Appendix B):

TABLE I. Solvation free energies $(\mathrm{kcal} / \mathrm{mol})$ for selected molecules and ions in water calculated with this model using a dielectric function $\epsilon=\epsilon(\rho)$ determined by the electron density (Ref. 13) and $\epsilon=\epsilon\left(\gamma\left(\mathbf{R}_{\mathbf{I}}\right)\right)$ determined by the atomic positions with $\beta=2.4$ (see text). Experimental values (Refs. 48 and 49)and results from PCM [obtained with the polarizable continuum model as implemented in GAUSSIAN 03 (Refs. 9 and 27] are also shown.

\begin{tabular}{lcccc}
\hline \hline & Expt. & $\epsilon=\epsilon(\rho)$ & $\epsilon=\epsilon(\gamma)$ & PCM \\
\hline $\mathrm{H}_{2} \mathrm{O}$ & -6.3 & -8.4 & -8.8 & -5.4 \\
$\mathrm{CH}_{3} \mathrm{CONH}_{2}$ & -9.7 & -10.5 & -8.0 & -4.6 \\
$\mathrm{CH}_{3} \mathrm{NH}_{3}^{+}$ & -73 & -81.0 & -81.9 & -65.1 \\
$\mathrm{NO}_{3}^{-}$ & -65 & -57.8 & -60.6 & -62.6 \\
$\mathrm{Cl}^{-}$ & -75 & -66.9 & $-68.6^{\mathrm{a}}$ & -72.6 \\
\hline \hline
\end{tabular}

${ }^{\mathrm{a}}$ The van der Waals radius for ionic chlorine was set equal to $2.059 \AA$ (from Ref. 47).

$$
\frac{\partial E_{H}}{\partial \mathbf{R}_{\mathbf{I}}}=-\frac{1}{8 \pi} \int \frac{\partial \epsilon\left(\mathbf{R}_{\mathbf{I}}\right)}{\partial \mathbf{R}_{\mathbf{I}}}(\nabla \phi(\mathbf{r}))^{2} d \mathbf{r}+\int \phi(\mathbf{r}) \frac{\partial \rho_{\mathrm{tot}}(\mathbf{r})}{\partial \mathbf{R}_{\mathbf{I}}} d \mathbf{r} .
$$

The computation of the first term on the right is straightforward since we know, from Eqs. (9) and (10), the explicit dependence of $\epsilon$ on $\mathbf{R}_{\mathbf{I}}$,

$$
\begin{aligned}
\frac{\partial \epsilon\left(\mathbf{R}_{\mathbf{I}}\right)}{\partial \tau_{I}}(\mathbf{r})= & 2 \beta\left(\epsilon_{\infty}-1\right) \\
& \times\left(\frac{\tau-\tau_{0}}{R}\right) \frac{e^{-\left(R-R_{\mathrm{vdw}}^{I}\right)}\left(\Sigma_{I} e^{-\left(R-R_{\mathrm{vdw}}^{I}\right)}\right)^{2 \beta-1}}{\left[1+\left(\Sigma_{I} e^{-\left(R-R_{\mathrm{vdw}}^{I}\right)}\right)^{2 \beta}\right]^{2}},
\end{aligned}
$$

with $\tau$ a generic coordinate $x, y$, or $z, \mathbf{R}_{\mathbf{I}}=\left(x_{0}, y_{0}, z_{0}\right)$ and $R$ $=\left|\mathbf{r}-\mathbf{R}_{\mathbf{I}}\right|$.

On the other hand, if $\phi(\mathbf{r})$ is transformed to Fourier space so that $\phi(\mathbf{r})=\Sigma_{\mathbf{G}} \widetilde{\phi}(\mathbf{G}) e^{i \mathbf{G r}}$ (see next section), the second term in Eq. (11) can be evaluated as

$$
\int \phi(\mathbf{r}) \frac{\partial \rho_{\mathrm{tot}}(\mathbf{r})}{\partial \mathbf{R}_{\mathbf{I}}} d \mathbf{r}=-\Omega \sum_{\mathbf{G}} i \mathbf{G} \widetilde{\phi}^{*}(\mathbf{G}) \widetilde{\rho}_{I}(\mathbf{G}) e^{-i \mathbf{G R}_{\mathbf{I}}}
$$

with $\tilde{\rho}_{I}(\mathbf{G})$ the form factor of the ionic densities, $\rho_{I}\left(\mathbf{r}-\mathbf{R}_{\mathbf{I}}\right)$ $=\Sigma_{G} \tilde{\rho}_{I}(\mathbf{G}) e^{-i \mathbf{G r}} e^{-i \mathbf{G} \mathbf{R}_{\mathbf{I}}}$.

To ensure the conservation of the total energy during the molecular dynamics simulations, the contributions given in Eqs. (12) and (13) must take the place of the derivative of the Hartree energy in the absence of the dielectric,

$$
\frac{\partial E_{H}}{\partial \mathbf{R}_{\mathbf{I}}}=-4 \pi \Omega \sum_{\mathbf{G}} i \mathbf{G}\left(\frac{\widetilde{\boldsymbol{\rho}}^{*}(\mathbf{G})}{G^{2}}\right) \widetilde{\rho}_{I}(\mathbf{G}) e^{-i \mathbf{G} \mathbf{R}_{\mathbf{I}}}
$$

\section{F. The multigrid scheme}

In standard plane-wave codes based on real space grids, the electrostatic potential $\phi(\mathbf{r})$ is obtained from the Poisson equation (2), which can be efficiently inverted with the use of fast Fourier transforms (FFTs). Both the total charge density $\rho_{\text {tot }}(\mathbf{r})$ and $\phi(\mathbf{r})$ can be expanded in plane waves,

$$
\rho_{\text {tot }}(\mathbf{r})=\sum_{\mathbf{G}} \widetilde{\rho}(\mathbf{G}) e^{i \mathbf{G r}}, \quad \phi(\mathbf{r})=\sum_{\mathbf{G}} \widetilde{\phi}(\mathbf{G}) e^{i \mathbf{G r}} .
$$

Replacing into the Poisson equation $\nabla^{2} \phi=-4 \pi \rho_{\text {tot }}$ and equating coefficients,

$$
\mathbf{G}^{2} \widetilde{\phi}(\mathbf{G})=4 \pi \widetilde{\rho}(\mathbf{G}), \quad \phi(\mathbf{r})=\sum_{\mathbf{G}} \frac{4 \pi}{\mathbf{G}^{2}} \widetilde{\rho}(\mathbf{G}) e^{i \mathbf{G r}} .
$$

Unfortunately, the Poisson equation in the presence of an arbitrary dielectric [Eq. (3)] cannot be addressed in the same way, and an alternative numerical scheme is required. To that end, we have implemented from scratch a sixth-order multigrid code, ${ }^{32}$ which solves in real space the Poisson equation with nonconstant coefficients and periodic boundary conditions. Equation (3) can be rewritten as 


$$
\frac{\partial \epsilon}{\partial x} \frac{\partial \phi}{\partial x}+\frac{\partial \epsilon}{\partial y} \frac{\partial \phi}{\partial y}+\frac{\partial \epsilon}{\partial z} \frac{\partial \phi}{\partial z}+\epsilon\left(\frac{\partial^{2} \epsilon}{\partial x^{2}}+\frac{\partial^{2} \epsilon}{\partial y^{2}}+\frac{\partial^{2} \epsilon}{\partial z^{2}}\right)=-4 \pi \rho
$$

This equation is developed in finite differences, expanding the derivatives of $\phi$ and $\epsilon$ to sixth-order according to the following relations for the gradient and the Laplacian:

$$
\begin{gathered}
\frac{\partial f(\mathbf{r})}{\partial \tau}=\frac{1}{h} \sum_{n=-3}^{3} \alpha_{n} u_{i+n}+O\left(h^{7}\right), \\
\frac{\partial^{2} f(\mathbf{r})}{\partial \tau^{2}}=\frac{1}{h^{2}} \sum_{n=-3}^{3} \beta_{n} u_{i+n}+O\left(h^{7}\right),
\end{gathered}
$$

where $\tau$ is a generic coordinate $x, y$, or $z ; h$ is the grid spacing in the direction $\tau$, and $u$ is the discretization of a continuous function $f(\mathbf{r})$, representing $\phi(\mathbf{r}), \rho(\mathbf{r})$, or $\epsilon(\mathbf{r}) . u_{i}$ refers to $u$ evaluated at a mesh point associated with $\mathbf{r}$, while $u_{i+n}$ corresponds to a neighboring point $n$ positions to the right in the direction $\tau$ (if $n<0, u_{i+n}$ is located to the left of $\left.u_{i}\right) .{ }^{33}$

In the case of $\epsilon(\mathbf{r})=1$ for all $\mathbf{r}$, this method provides a solution for $\phi(\mathbf{r})$ which is indistinguishable from the one obtained with FFT. It also demonstrated an excellent performance when tried on functions with nonconstant coefficients and oscillation frequencies comparable to those of interest. For example, for $\phi=e^{-a r}$ and $\epsilon=e^{-b r}(0.5<a, b<2.0)$ the relative error in $\phi(\mathbf{r})$ was less than $10^{-4}$ in a mesh of 80 $\times 80 \times 80$ points.

At the initial steps of a molecular dynamics simulation, the convergence of the potential may require 15-30 multigrid cycles. Given the self-consistent nature of the procedure, however, after a few time steps the number of cycles necessary to reach convergence is typically decreased to less than five. Even so, the multigrid algorithm is still significantly more expensive than FFTs. Propitiously, multigrid methods can be adapted to any kind of boundary conditions, and this feature can be exploited to reduce the size of the mesh involved. To implement this idea, a region in the supercellpreferably the slab-must remain inaccessible to the solvent so that $\epsilon(\mathbf{r})=1$ within it. In practice, the dielectric function of Eq. (7) or Eq. (10) is not diffuse enough as to encompass all the volume of the slab-if it were, the solvation effect would fade at the molecular boundaries-so the solvent occupies the interstitial space left by the atomic structure [Fig. 3(a)]. This is inconvenient not only because it increases the numerical complexity of the problem, but also because it is not physical, i.e., the solvent does not penetrate the atomic structure of the surface. A simple device to exclude the solvent from the solid interspaces is to modify $\gamma(\mathbf{r})$ in the following way:

$$
\gamma(\mathbf{r})= \begin{cases}\sum_{I} e^{-\left(\left|\mathbf{r}-\mathbf{R}_{\mathbf{I}}\right|-R_{\mathrm{vdw}}^{I}\right)}, & z_{I}>z_{\mathrm{lim}} \\ \sum_{I} e^{-z-z_{I}\left|z-z_{I}\right|\left(\left|\mathbf{r}-\mathbf{R}_{\mathbf{I}}\right|-R_{\mathrm{vdw}}^{I}\right)}, & z_{I} \leq z_{\mathrm{lim}},\end{cases}
$$

where $z_{I}$ is the $z$-component of $\mathbf{R}_{\mathbf{I}}$ (we recall $z$ is the coordinate perpendicular to the surface; it is zero at the bottom of the unit cell and maximum at the top). The factor $\left(z-z_{I}\right) / \mid z$

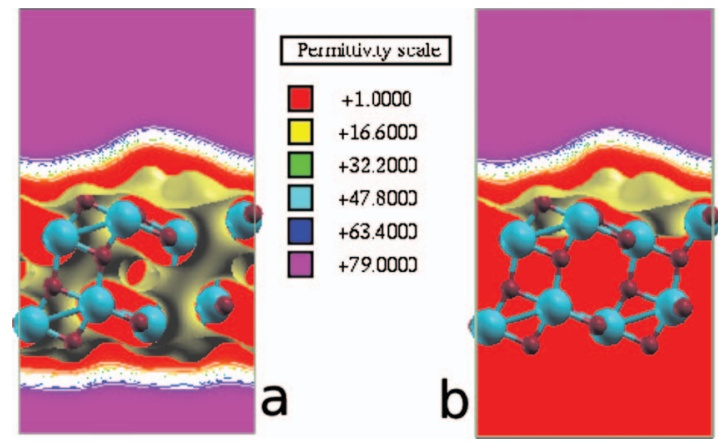

FIG. 3. Contour plot of the dielectric function $\epsilon(\mathbf{r})$ in a supercell containing a four layer slab representing the anatase (101) face of $\mathrm{TiO}_{2}$. An isosurface corresponding to $\epsilon(\mathbf{r})=1.4$ is displayed in yellow. In (a) the solvent percolates through the surface, whereas in (b) it is excluded from the slab by virtue of the artifact given in relation (19).

$-z_{I} \mid$ produces a rapid increase in $\gamma(\mathbf{r})$ underneath the atom $I$, which saturates the value of $\epsilon(\mathbf{r})$ for all atoms $I$ located below $z_{\text {lim. }}$. Thus, only the upper face of the slab is in contact with the solution, the dielectric function becoming equal to one throughout the lower section of the supercell. Figure 3(b) depicts $\epsilon(\mathbf{r})$ when $z_{\text {lim }}$ is chosen equal to the $z$ coordinate of an ion belonging to the second layer.

\section{APPLICATIONS: MOLECULAR DYNAMICS AT THE $\mathrm{TiO}_{2}$ INTERFACE}

It is well known that surface groups of most inorganic oxides ionize in solution, exhibiting the following equilibria:

$$
\begin{aligned}
& \mathrm{M}-\mathrm{O}^{z}+\mathrm{H}_{3} \mathrm{O}^{+} \Leftrightarrow \mathrm{M}-\mathrm{OH}^{z+1}+\mathrm{H}_{2} \mathrm{O} \Leftrightarrow \mathrm{M}-\mathrm{OH}_{2}^{z+2} \\
& +\mathrm{HO}^{-},
\end{aligned}
$$

with $z$ the surface group charge, which can be negative, positive, or zero depending on the nature of the oxide. ${ }^{21}$ Understanding the acid-base behavior resulting from these equilibria is crucial in almost every application of these materials in solution. Isoelectric points of many oxides have been known for years $;{ }^{34}$ however, it is very difficult to probe the surface of bulk materials or nanoparticles in solution, and most of the data collected correspond to the average behavior of the different surfaces exposed in a given experiment. More recently, researchers have sought to take advantage of DFT to establish the degree of dissociation and protonation at different titania-water interfaces with an explicit representation of the solvent. ${ }^{35-37}$ For the reasons already discussed, such an approach is costly and has been employed only in a limited number of cases. In what follows, we apply our continuum solvent model to characterize the hydrated (101) surface of the anatase structure of $\mathrm{TiO}_{2}$, which is possibly the most stable and abundant. ${ }^{4}$ The adsorption of $\mathrm{H}_{2} \mathrm{O}$ on perfect $\mathrm{TiO}_{2}$ surfaces in the gas phase has been thoroughly investigated using both experimental and theoretical approaches. ${ }^{38-43} \mathrm{In}$ the case of the (101) face of anatase, there is consensus in the fact that molecular adsorption of water is thermodynamically the most stable. Electronic structure calculations suggest that the difference between the two possible adsorption modesmolecular versus dissociated-is of nearly $10 \mathrm{kcal} / \mathrm{mol}^{41} \mathrm{We}$ have performed calculations in four layers slabs representing 
TABLE II. Adsorption energies for water on the anatase (101) surface in the gas phase and in solution in both the molecular and dissociative configurations at different coverages. Note that data in solution represent the energetic cost of exchanging implicit for explicit water. Values are given in kcal per $\mathrm{H}_{2} \mathrm{O}$ molecule.

\begin{tabular}{lccccc}
\hline \hline & \multicolumn{2}{c}{ Molecular } & & \multicolumn{2}{c}{ Dissociative } \\
\cline { 2 - 3 } \cline { 5 - 6 } & $\theta=0.25$ & $\theta=1$ & & $\theta=0.25$ & $\theta=1$ \\
\hline Gas phase & -19.4 & -17.8 & & -1.3 & -7.4 \\
Solution & $\cdots$ & -3.0 & & $\cdots$ & 6.6 \\
\hline \hline
\end{tabular}

the (101) surface of the anatase structure. As previously reported, our own calculations in vacuum summarized in Table II show that at different water coverages, the molecular pathway is the most favored. The adsorption energies from the liquid phase, given in the same table, indicate that molecular adsorption prevails also in the presence of the continuum solvent. It should be noted, though, that because of the dielectric embedding, the binding energy of $\mathrm{H}_{2} \mathrm{O}$ cannot be computed in a straightforward manner as in the gas phase: the values informed in Table II are energy differences between the explicit and implicit hydrations of the interface. In other words, the slightly negative number $(-3.0 \mathrm{kcal} / \mathrm{mol})$ corresponding to molecular adsorption is just the difference between the interaction of the surface with a water monolayer and with the implicit solvent and must not be misinterpreted in the sense that $\mathrm{TiO}_{2}$ affinity for water is weakened in solution. Such a small energy is indeed a quite remarkable result because it states that the continuum model retains the magnitude of the explicit water-oxide interaction. Moreover, the stabilization of up to $3 \mathrm{kcal} / \mathrm{mol}$ with respect to implicit hydration could be in part ascribed to the hydrogen bonding network arising within the monolayer, not accounted for in the continuum model. In the same way, the positive energy reported for dissociative adsorption does not imply that dissociation in solution is not exothermic, but it is just less exothermic than molecular hydration. The main result driven from these data is that the energy difference between the two kinds of adsorption mechanisms, $9.6 \mathrm{kcal} / \mathrm{mol}$, remains about the same as in the gas phase. In solution, however, dissociation is likely to occur, controlled by the $p \mathrm{H}$ of the medium (see below).

The quantitative effect of $p \mathrm{H}$ on the ionization of the surface is quite difficult to assess from first-principles simulations since a huge supercell would be needed to have a meaningful representation of the proton concentration in the system. In this preliminary study, we limit ourselves to examine the proton exchange between an adsorbed water molecule and a hydroxyl anion from the solution using molecular dynamics at $300 \mathrm{~K}$. This computational experiment is meant to provide a qualitative picture of the abstraction of a proton from the surface in the presence of $\mathrm{OH}^{-}$, illustrating at the same time the performance of the continuum solvent method. Solvation of hydrophilic surfaces often gives rise to structured contact layers in which the dielectric constant and other properties can significantly differ from those of bulk water, rendering unreliable the use of an implicit solvent model. In such cases, explicit consideration of a few water

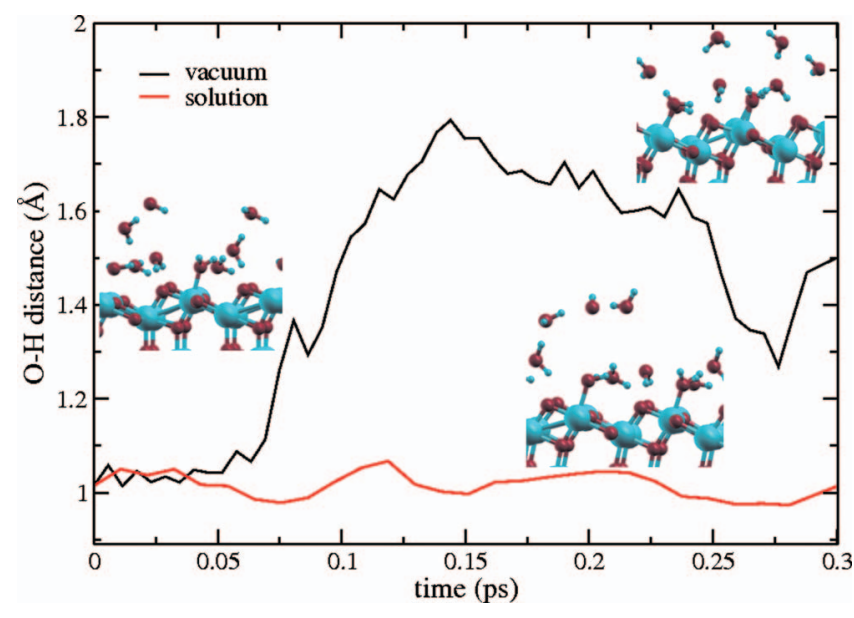

FIG. 4. Interatomic $\mathrm{O}_{a}-\mathrm{H}_{a}$ distance during molecular dynamics simulations of a water bilayer adsorbed on titania and in contact with an $\mathrm{OH}^{-}$ion in vacuum and in a continuum solvent. $\mathrm{O}_{a}$ and $\mathrm{H}_{a}$ are atoms of a water molecule $\mathrm{H}$-bonded to the $\mathrm{OH}^{-}$group at the beginning of the simulation. Proton transfer is seen in vacuum but not in solution. The starting geometry is shown on the left, while the upper and lower figures on the right depict the atomic structures in vacuum and in solution for the final time steps.

molecules belonging to the first solvation layers could be important. Recent molecular dynamics simulations at the $\mathrm{TiO}_{2}$-water interface using classical potentials have shown that the properties of the interface converge very rapidly to those of the bulk phase beyond the second adsorbed layer. $^{44,45}$ In light of this, we include in our system eight water molecules making up the first two solvation layers, which should be enough to retrieve a qualitatively correct representation of the interface within the scope of the present analysis. The inset of Fig. 4 shows the initial and final structures corresponding to two molecular dynamics simulations, one in vacuum and the other in solution, started from the same geometry and with identical computational parameters. In the initial configuration an $\mathrm{OH}^{-}$group exhibits a $\mathrm{H}$-bond with a water molecule of the second hydration layer. Figure 4 presents the intramolecular $\mathrm{O}_{a}-\mathrm{H}_{a}$ distance, where $\mathrm{H}_{a}$ is the proton involved in the $\mathrm{H}$-bond with the hydroxide. Early in the gas phase simulation, the covalent $\mathrm{O}_{a}-\mathrm{H}_{a}$ bond in $\mathrm{H}_{2} \mathrm{O}$ is disrupted, and the $\mathrm{H}_{a}$ atom is transferred to the neighboring $\mathrm{OH}^{-}$group, triggering a Grotthuss-like exchange of protons to leave an hydroxyl function on the surface. This is not at all surprising but is just reflecting the fact that a negative charge would rather localize on the oxide surface than on an hydroxide ion exposed to the gas phase. In the continuum solvent, this trend is reverted. The negatively charged hydroxide is stabilized in the polar environment and remains as part of the hydrogen-bonding network.

Had the simulation been started from a random configuration in the presence of the implicit solvent, the $\mathrm{OH}^{-}$anion might have explored the supercell for several picoseconds without ever reacting with the water molecules of the contact layers. In the absence of the solvent, instead, the unscreened interaction between the hydroxide and the $\mathrm{H}_{2} \mathrm{O}$ molecules leads to an immediate reaction, and the final result is the hydroxylation of the $\mathrm{TiO}_{2}$ surface, as already commented. This distinctive behavior is displayed in Fig. 5, which presents the $\mathrm{O}_{b}-\mathrm{H}_{a}$ distances for two simulations in vacuum 


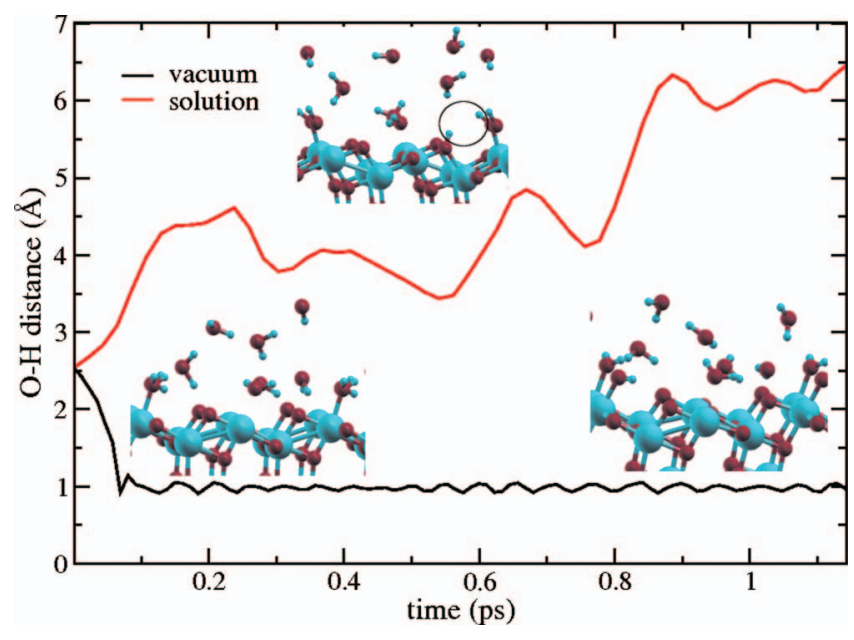

FIG. 5. Interatomic distance $\mathrm{O}_{b}-\mathrm{H}_{a}$ during the molecular dynamics simulations of a water bilayer adsorbed on titania in the presence of an hydroxide ion initially situated $2.5 \AA$ away from the closest water molecule. $\mathrm{O}_{b}$ is the oxygen atom of the hydroxide, and $\mathrm{H}_{a}$ is one of the nearest $\mathrm{H}_{2} \mathrm{O}$ protons, which are rapidly exchanged during the gas phase dynamics. In solution, instead, the interaction between the $\mathrm{OH}^{-}$ion and the interface is efficiently screened. The starting geometry is shown on the left, while the figures on the center and on the right depict the atomic structures after 1 ps of dynamics in solution and in vacuum, respectively. A spontaneously dissociated water, enclosed in a black trace, is observed in the continuum solvent.

and in solution, departing from the same configuration, with $\mathrm{O}_{b}$ the oxygen atom of the hydroxide and $\mathrm{H}_{a}$ the atom abstracted from the hydration layer in vacuum. The observed contrast between the two dynamics evinces how the dielectric medium stabilizes the hydroxide in the liquid phase. Interestingly enough, dissociation of an adsorbed water molecule takes place during the simulation in the implicit solvent (see inset of Fig. 5). Spontaneous dissociation of water has never been observed in simulations of the stoichiometric (101) anatase surface with up to three hydration monolayers, ${ }^{5,36}$ suggesting that the continuum solvent may play a significant role even when several water layers are considered. The use of this kind of methodology in combination with weighted importance sampling techniques (e.g., umbrella sampling) ${ }^{46}$ could provide estimates for the acidbase equilibrium constants corresponding to different oxide surfaces and phases. We believe this direction, even though beyond the scope of the present work-with an emphasis on the methodological conception-aims to a very appealing ground for future research.

\section{CLOSING REMARKS}

We have shown that a dielectric medium defined as a function of the self-consistent charge density provokes a strong response in the effective potential, which in solidliquid systems may spoil the convergence of the CarParrinello electronic dynamics. Such a response can be avoided with a dielectric based on a non-self-consistent charge, preserving in this way the potential and allowing for conservative molecular dynamics simulations. This approach is equivalent to have a position-dependent permittivity, and therefore a new term in the ionic forces must be considered.

The methodology presented here is a powerful instrument to explore the thermodynamics and the reactivity of surfaces and nanoparticles in solution. Replacement of the solvent molecules by a dielectric continuum may neglect the structural features of the liquid phase, but it does capture the essential polarization effect of the medium. This is manifested, for instance, in the charge of the hydroxyl group: if an additional electron is added to a neutral system consisting of a slab plus a (distant) $\mathrm{OH}$ moiety in vacuum, a significant portion of the charge (about $0.5 e$ ) flows to the solid phase. Noticeably, in the presence of the solvent, the excess electron spontaneously localizes on the hydroxyl.

A compelling application for this continuum solvent scheme, as well as a natural continuation of this work, would be the characterization of the adsorption energies and geometries of water and other species on the different surfaces of titanium dioxide. We deem especially worthwhile the calculation of the reaction energies for the kind of equilibria mentioned above, e.g., $\mathrm{Ti}-\mathrm{OH}_{2}+\mathrm{OH}^{-} \Leftrightarrow \mathrm{Ti}-\mathrm{OH}^{-}+\mathrm{H}_{2} \mathrm{O}$, as a function of the surface structure. Work in this direction is now in progress. At this point it should be noted that it would not be feasible to have an estimate of these quantities without the solvation model: in the absence of the dielectric, the interaction between the surface and the $\mathrm{OH}^{-}$ion (or between the charged slab and the water molecule) is extremely dependent on the distance separating them, and therefore it is not possible to establish unequivocally the energies for reactants and products. When the dielectric is included, the long range interaction between charged and polar fragments is efficiently screened, and the total energy of the system becomes independent of the position of the molecule (or the ion) with respect to the slab. This property makes it possible to evaluate reaction energies on periodic surfaces in solution, which could not be calculated by other means, except perhaps with extensive molecular dynamics simulations. Aside from these, the scheme presented here would be particularly useful to assess the role of the solvent in a great diversity of problems in surface chemistry, including the effects on structure, on vibrational frequencies, or on charge transfer phenomena, among many others.

\section{ACKNOWLEDGMENTS}

We feel indebted to Jean-Luc Fattebert, Oswaldo Dieguez, Ismailia Dabo, Nicola Marzari, and Patu Groisman for precious advice and enlightening discussions. This study was financially supported by CONICET (Grant No. PIP5220) and by the Agencia Nacional de Promoción Científica y Tecnológica (Grant No. PICT 06-33581). V.M.S. acknowledges CONICET for a doctoral fellowship.

\section{APPENDIX A: FUNCTIONAL DERIVATIVE OF $\boldsymbol{v}_{f}$}

Following Parr and Yang, ${ }^{30}$ the functional derivative of $E_{H}[\rho]$ with respect to $\rho(\mathbf{r})$, which we will denote as $\delta E_{H} / \delta \rho$, is defined by the relation 


$$
\lim _{\lambda \rightarrow 0}\left[\frac{E_{H}(\rho+\lambda f)-E_{H}(\rho)}{\lambda}\right]=\int \frac{\delta E_{H}}{\delta \rho} f(\mathbf{r}) d \mathbf{r},
$$

where $f(\mathbf{r})$ is arbitrary. From the definition of $E_{H}[\rho]$ we can write

$$
\begin{aligned}
& 2 E_{H}(\rho+\lambda f)-2 E_{H}(\rho) \\
& \quad=\int \phi_{\rho+\lambda f}(\rho+\lambda f) d \mathbf{r}-\int \phi_{\rho} \rho d \mathbf{r} \\
& \quad=\int \phi_{\rho+\lambda f} \rho d \mathbf{r}+\lambda \int \phi_{\rho+\lambda f} f d \mathbf{r}-\int \phi_{\rho} \rho d \mathbf{r} \\
& \quad=\lambda \int \phi_{\rho+\lambda f} f d \mathbf{r}+\int\left(\phi_{\rho+\lambda f}-\phi_{\rho}\right) \rho d \mathbf{r} .
\end{aligned}
$$

In the last term above $\rho$ can be rewritten using Eq. (3), and the resulting expression can be integrated by parts (from now on we omit $d \mathbf{r}$ from the integrand for conciseness),

$$
\begin{aligned}
& \int\left(\phi_{\rho+\lambda f}-\phi_{\rho}\right) \rho \\
&=-\frac{1}{4 \pi} \int\left(\phi_{\rho+\lambda f}-\phi_{\rho}\right) \nabla\left(\epsilon_{\rho} \nabla \phi_{\rho}\right) \\
&=\frac{1}{4 \pi} \int \nabla \phi_{\rho+\lambda f} \epsilon_{\rho} \nabla \phi_{\rho}-\frac{1}{4 \pi} \int \nabla \phi_{\rho} \epsilon_{\rho} \nabla \phi_{\rho} \\
&=\frac{1}{4 \pi} \int \nabla \phi_{\rho+\lambda f}\left(\epsilon_{\rho}-\epsilon_{\rho+\lambda f}\right) \nabla \phi_{\rho} \\
& \quad+\frac{1}{4 \pi} \int \nabla \phi_{\rho+\lambda f} \epsilon_{\rho+\lambda f} \nabla \phi_{\rho}-\frac{1}{4 \pi} \int \nabla \phi_{\rho} \epsilon_{\rho} \nabla \phi_{\rho} \\
&=\frac{1}{4 \pi} \int \nabla \phi_{\rho+\lambda f}\left(\epsilon_{\rho}-\epsilon_{\rho+\lambda f}\right) \nabla \phi_{\rho}+\int(\rho+\lambda f) \phi_{\rho} \\
&-\frac{1}{4 \pi} \int \epsilon_{\rho}\left(\nabla \phi_{\rho}\right)^{2} .
\end{aligned}
$$

To arrive to the last expression, $\epsilon_{\rho+\lambda f}$ was added and subtracted in the third equality, and then the second term was integrated by parts. This result can now be inserted in Eq. (A2),

$$
\begin{aligned}
2 E_{H}(\rho+\lambda f)-2 E_{H}(\rho) & \\
= & \lambda \int \phi_{\rho+\lambda f} f+\frac{1}{4 \pi} \int \nabla \phi_{\rho+\lambda f}\left(\epsilon_{\rho}-\epsilon_{\rho+\lambda f}\right) \nabla \phi_{\rho} \\
& +\lambda \int f \phi_{\rho}+\int \rho \phi_{\rho}-\frac{1}{4 \pi} \int \epsilon_{\rho}\left(\nabla \phi_{\rho}\right)^{2} \\
= & \lambda \int\left(\phi_{\rho+\lambda f}+\phi_{\rho}\right) f-\frac{1}{4 \pi} \int \nabla \phi_{\rho+\lambda f}\left(\epsilon_{\rho+\lambda f}-\epsilon_{\rho}\right) \nabla \phi_{\rho} .
\end{aligned}
$$

Dividing Eq. (A3) by $2 \lambda$ and taking the limit $(\lambda \rightarrow 0)$, we get to the final outcome,

$$
\begin{aligned}
& \lim _{\lambda \rightarrow 0}[\left.\frac{E_{H}(\rho+\lambda f)-E_{H}(\rho)}{\lambda}\right] \\
&=\lim _{\lambda \rightarrow 0}\left[\frac{1}{2} \int\left(\phi_{\rho+\lambda f}+\phi_{\rho}\right) f\right. \\
&\left.\quad-\frac{1}{8 \pi} \int \nabla \phi_{\rho+\lambda f} \nabla \phi_{\rho} \frac{\left(\epsilon_{\rho+\lambda f}-\epsilon_{\rho}\right)}{\lambda}\right] \\
&=\int \phi_{\rho} f-\frac{1}{8 \pi} \int \nabla \phi_{\rho} \nabla \phi_{\rho} \frac{\partial \epsilon}{\partial \rho} f \\
&=\int\left(\phi_{\rho}-\frac{1}{8 \pi}\left(\nabla \phi_{\rho}\right)^{2} \frac{\partial \epsilon}{\partial \rho}\right) f(\mathbf{r}) d \mathbf{r} .
\end{aligned}
$$

By comparison with Eq. (A1), it is easy to see that $\delta E_{H} / \delta \rho=\phi_{\rho}-(1 / 8 \pi)\left(\nabla \phi_{\rho}\right)^{2} \partial \epsilon / \partial \rho$.

\section{APPENDIX B: DERIVATIVE OF $E_{H}$ WITH RESPECT TO THE IONIC POSITIONS}

In the absence of a dielectric $(\epsilon=1)$, Eq. (15) can be inserted in the expression for the Hartree energy to give

$$
E_{H}=2 \pi \Omega \sum_{\mathbf{G}} \frac{|\widetilde{\rho}(\mathbf{G})|^{2}}{G^{2}},
$$

where $\tilde{\rho}(\mathbf{G})$ are the Fourier coefficients for the expansion of

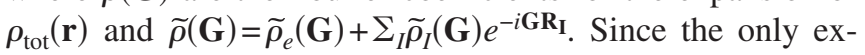
plicit dependence of $\tilde{\rho}(\mathbf{G})$ on $\left\{\mathbf{R}_{\mathbf{I}}\right\}$ is through the structure

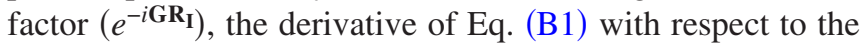
atomic positions is just

$$
\frac{\partial E_{H}}{\partial \mathbf{R}_{\mathbf{I}}}=-4 \pi \Omega \sum_{\mathbf{G}} i \mathbf{G}\left(\frac{\widetilde{\rho}^{*}(\mathbf{G})}{G^{2}}\right) \widetilde{\rho}_{I}(\mathbf{G}) e^{-i \mathbf{G} \mathbf{R}_{\mathbf{I}}} .
$$

In the presence of a dielectric medium determined by the ionic coordinates, Eq. (B1) does not hold. To calculate $\partial E_{H} / \partial \mathbf{R}_{\mathbf{I}}$ we replace $\epsilon[\rho]$ for $\boldsymbol{\epsilon}\left(\mathbf{R}_{\mathbf{I}}\right)$ in Eq. (4) and derivate

$$
\begin{aligned}
\frac{\partial E_{H}}{\partial \mathbf{R}_{\mathbf{I}}}= & \frac{1}{8 \pi} \int \frac{\partial \boldsymbol{\epsilon}\left(\mathbf{R}_{\mathbf{I}}\right)}{\partial \mathbf{R}_{\mathbf{I}}}(\nabla \phi(\mathbf{r}))^{2} d \mathbf{r} \\
& +\frac{1}{8 \pi} \int \boldsymbol{\epsilon}\left(\mathbf{R}_{\mathbf{I}}\right) \frac{\partial(\nabla \phi(\mathbf{r}))^{2}}{\partial \mathbf{R}_{\mathbf{I}}} d \mathbf{r} .
\end{aligned}
$$

The second term on the right member can be further developed as follows:

$$
\begin{aligned}
\frac{1}{8 \pi} \int \epsilon\left(\mathbf{R}_{\mathbf{I}}\right) \frac{\partial(\nabla \phi(\mathbf{r}))^{2}}{\partial \mathbf{R}_{\mathbf{I}}} d \mathbf{r} \\
=\frac{2}{8 \pi} \int \epsilon\left(\mathbf{R}_{\mathbf{I}}\right) \nabla \phi(\mathbf{r}) \nabla \frac{\partial \phi(\mathbf{r})}{\partial \mathbf{R}_{\mathbf{I}}} d \mathbf{r} \\
=-\frac{1}{4 \pi} \int \nabla \cdot\left[\epsilon\left(\mathbf{R}_{\mathbf{I}}\right) \nabla \phi(\mathbf{r})\right] \frac{\partial \phi(\mathbf{r})}{\partial \mathbf{R}_{\mathbf{I}}} d \mathbf{r} \\
=\int \rho_{\text {tot }}(\mathbf{r}) \frac{\partial \phi(\mathbf{r})}{\partial \mathbf{R}_{\mathbf{I}}} d \mathbf{r},
\end{aligned}
$$

where we have integrated by parts and used Eq. (3). Then, it is possible to rewrite Eq. (B3), 


$$
\frac{\partial E_{H}}{\partial \mathbf{R}_{\mathbf{I}}}=\frac{1}{8 \pi} \int \frac{\partial \boldsymbol{\epsilon}\left(\mathbf{R}_{\mathbf{I}}\right)}{\partial \mathbf{R}_{\mathbf{I}}}(\nabla \phi(\mathbf{r}))^{2} d \mathbf{r}+\int \rho_{\mathrm{tot}}(\mathbf{r}) \frac{\partial \phi(\mathbf{r})}{\partial \mathbf{R}_{\mathbf{I}}} d \mathbf{r} .
$$

On the other hand, the derivation of the general expression for the Hartree energy leads to

$$
\frac{\partial E_{H}}{\partial \mathbf{R}_{\mathbf{I}}}=\frac{1}{2} \int \rho_{\mathrm{tot}}(\mathbf{r}) \frac{\partial \phi(\mathbf{r})}{\partial \mathbf{R}_{\mathbf{I}}} d \mathbf{r}+\frac{1}{2} \int \phi(\mathbf{r}) \frac{\partial \rho_{\mathrm{tot}}(\mathbf{r})}{\partial \mathbf{R}_{\mathbf{I}}} d \mathbf{r} .
$$

Equating Eqs. (B5) and (B6) we find the following relation:

$$
\begin{aligned}
\int \rho_{\mathrm{tot}}(\mathbf{r}) \frac{\partial \phi(\mathbf{r})}{\partial \mathbf{R}_{\mathbf{I}}} d \mathbf{r}= & -\frac{1}{4 \pi} \int \frac{\partial \epsilon\left(\mathbf{R}_{\mathbf{I}}\right)}{\partial \mathbf{R}_{\mathbf{I}}}(\nabla \phi(\mathbf{r}))^{2} d \mathbf{r} \\
& +\int \phi(\mathbf{r}) \frac{\partial \rho_{\mathrm{tot}}(\mathbf{r})}{\partial \mathbf{R}_{\mathbf{I}}} d \mathbf{r} .
\end{aligned}
$$

Ultimately, replacing Eq. (B7) with Eq. (B5), we obtain the final result,

$$
\frac{\partial E_{H}}{\partial \mathbf{R}_{\mathbf{I}}}=-\frac{1}{8 \pi} \int \frac{\partial \boldsymbol{\epsilon}\left(\mathbf{R}_{\mathbf{I}}\right)}{\partial \mathbf{R}_{\mathbf{I}}}(\nabla \phi(\mathbf{r}))^{2} d \mathbf{r}+\int \phi(\mathbf{r}) \frac{\partial \rho_{\mathrm{tot}}(\mathbf{r})}{\partial \mathbf{R}_{\mathbf{I}}} d \mathbf{r} .
$$

${ }^{1}$ See in particular: E. A. Carter, in "Challenges in Theoretical Chemistry," special issue of Science 321 (5890), 800 (2008); G. J. Kroes, in "Challenges in Theoretical Chemistry," special issue of ibid. 321 (5890), 794 (2008).

${ }^{2}$ L. W. Bruch, R. D. Diehl, and J. A. Venables, Rev. Mod. Phys. 79, 1381 (2007).

${ }^{3}$ A. Nilsson and L. G. M. Pettersson, Surf. Sci. Rep. 55, 49 (2004).

${ }^{4}$ U. Diebold, Surf. Sci. Rep. 48, 53 (2003).

${ }^{5}$ It is possible to find in the literature a few number of studies in which one or several layers of water molecules are incorporated to represent the solvent. See, for example, A. Tilocca and A. Selloni, Langmuir 20, 8379 (2004); A. B. Mukhopadhyay, C. B. Musgrave, and J. Fdez Sanz, J. Am. Chem. Soc. 130, 11996 (2008).

${ }^{6}$ C. J. Cramer and D. G. Truhlar, Chem. Rev. (Washington, D.C.) 99, 2161 (1999).

${ }^{7}$ I. N. Levine, Quantum Chemistry (Prentice-Hall, New Jersey, 2000).

${ }^{8}$ T. Schlick, Molecular Modeling and Simulation (Springer-Verlag, New York, 2002).

${ }^{9}$ J. Tomasi, B. Mennucci, and R. Cammi, Chem. Rev. (Washington, D.C.) 105, 2999 (2005)

${ }^{10}$ F. De Angelis, A. Sgamellotti, M. Cossi, N. Rega, and V. Barone, Chem. Phys. Lett. 328, 302 (2000).

${ }^{11}$ J.-L. Fattebert and F. Gygi, Int. J. Quantum Chem. 93, 139 (2003).

${ }^{12}$ H. M. Senn, P. M. Margi, R. Schmid, T. Ziegler, and P. Blöchl, J. Chem. Phys. 118, 1089 (2003).

${ }^{13}$ D. A. Scherlis, J.-L. Fattebert, F. Gygi, M. Cococcioni, and N. Marzari, J. Chem. Phys. 124, 074103 (2006).

${ }^{14}$ Arias and co-workers devised a form of DFT for the self-consistent embedding of quantum-mechanical systems in a dielectric medium. This approach has been applied to investigate the atomic and electronic structure of the $\mathrm{Cr}_{2} \mathrm{O}_{3}$ surface in solution by means of static calculations. See S. A. Petrosyan, A. A. Rigos, and T. A. Arias, J. Phys. Chem. B 109, 15436 (2005); S. A. Petrosyan, J.-F. Briere, D. Roundy, and T. A. Arias, Phys. Rev. B 75, 205105 (2007).

${ }^{15}$ J.-L. Fattebert and F. Gygi, J. Comput. Chem. 23, 662 (2002).

${ }^{16}$ D. A. Scherlis, J.-L. Fattebert, and N. Marzari, J. Chem. Phys. 124, 194902 (2006).
${ }^{17}$ W. H. Press, S. A. Teukolsky, W. T. Vetterling, and B. P. Flannery, Numerical Recipes in Fortran, 2nd ed. (Cambridge University Press, New York, 1992)

${ }^{18}$ W. L. Briggs, V. Emden Henson, and S. F. McCormick, A Multigrid Tutorial, 2nd ed. (SIAM, Philadelphia, 2000).

${ }^{19}$ U. Trottenberg, C. W. Oosterlee, and A. Schüller, Multigrid (Elsevier Academic Press, San Diego, 2001).

${ }^{20}$ T. Hiemstra, P. Venema, and W. H. Van Riemsdijk, J. Colloid Interface Sci. 184, 680 (1996).

${ }^{21}$ J.-P. Jolivet, Metal Oxide Chemistry and Synthesis (Wiley, Chichester, 2000).

${ }^{22}$ S. Baroni, A. Dal Corso, S. de Gironcoli, P. Giannozzi, C. Cavazzoni, G. Ballabio, S. Scandolo, G. Chiarotti, P. Focher, A. Pasquarello, K. Laasonen, A. Trave, R. Car, N. Marzari, and A. Kokalj, http://www.quantumespresso.org/.

${ }^{23}$ J. P. Perdew, in Electronic Structure of Solids '91, edited by P. Ziesche and H. Eschrig (Akademie-Verlag, Berlin, 1991).

${ }^{24}$ D. Vanderbilt, Phys. Rev. B 41, 7892 (1990).

${ }^{25}$ D. Marx and J. Hutter, in Ab Initio Molecular Dynamics: Theory and Implementation, Modern Methods and Algorithms of Quantum Chemistry, edited by J. Grotendorst (John Von Neumann Institute for Computing, Jülich, 2000).

${ }^{26}$ G. Galli and A. Pasquarello, in Computer Simulation in Chemical Physics, edited by M. P. Allen and D. J. Tildesley (Kluwer-Academic, Dordrecht, The Netherlands, 1993).

${ }^{27}$ M. Cossi, V. Barone, R. Cammi, and J. Tomasi, Chem. Phys. Lett. 255, 327 (1996).

${ }^{28}$ Of course, the mere omission of $V_{\epsilon}$ would not be an acceptable solution in molecular dynamics simulations since the energy conservation will be affected.

${ }^{29}$ The parameter $\beta$ is taken greater than 0.5. See Ref. 15 .

${ }^{30}$ R. G. Parr and W. Yang, Density-Functional Theory of Atoms and Molecules (Oxford University Press, New York, 1989).

${ }^{31}$ I. Dabo, E. Cancès, Y. Li, and N. Marzari, e-print arXiv:org/abs/ 0901.0096 .

${ }^{32}$ Multigrid methods solve elliptic partial differential equations by applying a classic finite-differences iterative technique in meshes of different sizes (in this case we adopt the Gauss-Seidel scheme). See Ref. 17 for an introduction and Ref. 18 or Ref. 19 for more specialized sources.

${ }^{33}$ The coefficients $\alpha_{n}$ and $\beta_{n}$ are given by $\alpha_{0}=0, \alpha_{1}=\frac{3}{4}, \alpha_{2}=-\frac{3}{20}, \alpha_{3}=\frac{1}{60}$, $\alpha_{-n}=-\alpha_{n}, \beta_{0}=-\frac{49}{18}, \beta_{1}=\frac{27}{18}, \beta_{2}=-\frac{27}{180}, \beta_{3}=\frac{2}{180}$, and $\beta_{-n}=\beta_{n}$.

${ }^{34}$ G. A. Parks, Chem. Rev. (Washington, D.C.) 65, 177 (1965).

${ }^{35}$ A. V. Bandura, D. G. Sykes, V. Shapovalov, T. N. Troung, J. D. Kubicki, and R. A. Evarestov, Langmuir 108, 7844 (2004).

${ }^{36}$ A. Tilocca and A. Selloni, J. Phys. Chem. B 108, 4743 (2004).

${ }^{37}$ M. L. Machesky, M. Pedota, D. J. Wesolowski, L. Vlcek, P. T. Cummings, J. Rosenqvist, M. K. Ridley, J. D. Kubicki, A. V. Bandura, N. Kumar, and J. O. Sofo, Langmuir 24, 12331 (2008).

${ }^{38}$ M. A. Henderson, Surf. Sci. 355, 151 (1996).

${ }^{39}$ R. Schaub, P. Thostrup, N. Lopez, E. Laegsgaard, I. Stensgaard, J. K. Norskov, and F. Besenbacher, Phys. Rev. Lett. 87, 266104 (2001).

${ }^{40} \mathrm{G}$. Li, L. Li, J. Boerio-Goates, and B. F. Woodfield, J. Am. Chem. Soc. 127, 8659 (2005).

${ }^{41}$ A. Vittadini, A. Selloni, F. P. Rotzinger, and M. Grätzel, Phys. Rev. Lett. 81, 2954 (1998).

${ }^{42}$ A. Tilocca and A. Selloni, J. Chem. Phys. 119, 7445 (2003).

${ }^{43}$ P. J. D. Lindan and C. Zhang, Phys. Rev. B 72, 075439 (2005).

${ }^{44}$ M. Předota, A. V. Bandura, P. T. Cummings, J. D. Kubicki, D. J. Wesolowski, A. A. Chialvo, and M. L. Machesky, J. Phys. Chem. B 108, 12049 (2004)

${ }^{45}$ A. Kornherr, D. Vogtenhuber, M. Ruckenbauer, R. Podloucky, and G. Zifferer, J. Chem. Phys. 121, 3722 (2004).

${ }^{46}$ G. M. Torrie and J. P. Valleau, J. Comput. Phys. 23, 187 (1977).

${ }^{47}$ J. K. Badenhoop and F. Weinhold, J. Chem. Phys. 107, 5422 (1997).

${ }^{48}$ C. J. Cramer and D. G. Truhlar, J. Am. Chem. Soc. 113, 8305 (1991).

${ }^{49}$ G. D. Hawkins, C. J. Cramer, and D. G. Truhlar, J. Phys. Chem. B 102, 3257 (1998). See tables included in supporting information. 